\title{
Bienestar subjetivo, percepción de esfuerzo, aprendizaje y diversión en el esquí náutico inclusivo
}

\author{
David SUÁREZ-IGLESIAS y José Gerardo VILLA-VICENTE \\ Universidad de León. España
}

(Recibido, 14 abril 2017; Aceptado, 18 mayo 2017)

RESUMEN: Este estudio investigó la percepción de bienestar subjetivo (BS), aprendizaje, diversión y esfuerzo en un curso de esquí náutico inclusivo. Cinco adultos con discapacidades físicas con edades comprendidas entre 45 y 63 años $(M=52.6, D E=7.7)$, procedentes de un Centro de Referencia Estatal de Discapacidad y Dependencia, participaron en un curso de seis sesiones de esquí náutico inclusivo (ENI) en el medio natural de un embalse. La metodología del curso siguió un enfoque basado en capacidades, en torno a un espectro de inclusión aplicado al esquí náutico (Suárez-Iglesias y SuárezGarcía, 2016). El BS de los participantes se midió antes y después del curso con el cuestionario Wellbeing Index (WHO-5) (WHO, 1998). En cada sesión, la percepción de aprendizaje y diversión se valoró con el instrumento del "semáforo", elaborado por Palao y Hernández (2012), mientras que la intensidad se evaluó a través de la escala de percepción subjetiva del esfuerzo de Borg (1982). Los resultados muestran que el BS aumentó tras la realización del curso, y en la mayoría de sesiones los participantes consideraron haber aprendido y haberse divertido mucho, percibiendo generalmente el esfuerzo entre "bastante ligero" y "algo duro". En definitiva, la práctica de ENI por parte de adultos institucionalizados que envecejen con discapacidades podría promover su BS y servir como una actividad física de tiempo libre que disfrutan, donde aprenden y que no requiere gran esfuerzo.

Palabras clave: Discapacidad, actividad física adaptada, deporte acuático, disfrute, enseñanza.

Subjective well-being, perceived effort, learning and fun in inclusive water skiing

\begin{abstract}
This study investigated the perception of subjective well-being (SW) and perceived effort, learning and fun in an inclusive water skiing (IWS) course. Five adults with physical disabilities between the ages of 45 and $63(\mathrm{M}=52.6, \mathrm{SD}=7.7)$ from a State Reference Centre for Severely Disabled People participated in a six-session IWS course in the natural environment of a reservoir. The methodology of the course followed an abilities-based approach, applying an inclusion spectrum to water skiing (Suárez-Iglesias and Suárez-García, 2016). Participants' SW was measured before and after the course with the Well-being Index (WHO-5) questionnaire (WHO, 1998). In each session, the perception of learning and fun was assesed with the "traffic light" instrument by Palao and Hernández (2012), while the subjective perception of effort was assessed through Borg's scale (1982). The results show that SW increased after completing the course, and in most sessions participants considered they learnt and had a lot of fun, generally perceiving the effort between "quite light" and "somewhat hard".
\end{abstract}


Eventually, the practice of IWS by institutionalized adults with disabilities may promote their SW and serve as a leisure-time physical activity they enjoy, where they learn and do not require much effort.

Keywords: Disability, adapted physical activity, water sport, enjoyment, teaching.

Correspondencia: David Suárez Iglesias, c/ Federico García Lorca, n²2, Bajo E, 24195, Villaobispo de las Regueras, Villaquilambre (León). Email: $\underline{\text { dsuai@unileon.es }}$

\section{Introducción}

Las personas que envejecen con limitaciones de movimiento son cada vez más numerosas y presentan niveles de actividad más bajos que la población general, siendo particularmente valioso promover un estilo de vida activo que influya positivamente sobre todos los niveles de funcionamiento (Rosenberg, Bombardier, Hoffman, y Belza, 2011). Los españoles que envejecen con discapacidades y reducida autonomía para las actividades de la vida diaria pasan más tiempo en ocio activo que sus pares jóvenes (Pagán-Rodríguez, 2014). Así, a pesar del conjunto multifactorial de barreras únicas que afectan al grado de participación en actividad física en tiempo libre (AFTL) de las personas con discapacidades físicas, las estrategias para elevar la AFTL a largo plazo son muy necesarias (Martin Ginis, Ma, LatimerCheung, y Rimmer, 2016). Una opción para las personas con discapacidades físicas es el ejercicio desarrollado en la naturaleza, del cual existe evidencia sobre su incidencia directa y positiva en el bienestar psicológico de sus practicantes (Bowler, Buyung-Ali, Knight, y Pullin, 2010; Pasanen, Tyrväinen, y Korpela, 2014), pudiendo facilitar el disfrute, aumentar la adherencia y ser realizado con menor percepción de esfuerzo (Gladwell, Brown, Wood, Sandercock, y Barton, 2013).

Asimismo, uno de los objetivos de participación en actividad adaptada, desde una perspectiva inclusiva, es obtener diversión (Hutzler y Sherrill, 2007). En concreto, el grado de diversión de las actividades físico-deportivo-recreativas en la naturaleza condiciona la participación activa y la adherencia (Palmi y Martín, 1997). Paralelamente, cuando se trata de motivar a las personas para ser físicamente activas en ambientes naturales, aquellas con discapacidad experimentan mayores restricciones debido a problemas de programación (Drakou, De Vreese, Lofthus, y Muscat, 2011). Por tanto, los profesionales deberían adaptar las actividades físicas a las diferentes capacidades e intereses de los destinatarios del programa (Hutzler, 2003), proporcionando ambientes de gran impacto donde la calidad del aprendizaje y la relevancia para el participante sean claves (O’Brien et al., 2011). Mientras, Malone, Barfield, y Brasher (2012) detectaron que la principal barrera para hacer ejercicio en personas activas con discapacidades físicas y enfermedades crónicas era percibir el ejercicio como duro y fatigante. Por ello, Rosenberg et al. (2011) recomiendan en personas que envejecen con movilidad reducida optar por actividades físicas de intensidad aminorada.

Recientemente, Suárez-Iglesias y Suárez-García (2016) propusieron el esquí náutico como medio de inclusión social para personas con discapacidad. La presencia de agua incrementa los beneficios saludables del ejercicio en el medio natural (Barton y Pretty, 2010), y autores como Lundberg, Bennett, y Smith (2011) han resaltado el impacto potencial de programas recreativos terapeúticos basados en actividades físicas acuáticas en la naturaleza, incluyendo el esquí náutico, sobre áreas de calidad de vida de veteranos de combate con discapacidades físicas adquiridas. Incluso el surf adaptado ha sido defendido como estrategia 
de promoción del bienestar que mejore el proceso de rehabilitación de personas con discapacidades (Lopes, 2015).

En suma, el objetivo de este estudio fue conocer el impacto de un curso intensivo de esquí náutico inclusivo (en lo sucesivo, ENI) en los adultos con discapacidades físicas participantes, valorando los siguientes aspectos: a) el efecto sobre el bienestar psicológico subjetivo de realizar el curso, b) el aprendizaje, diversión y esfuerzo percibidos en el curso.

\section{Método}

\section{Participantes}

Participaron 5 adultos procedentes de un centro de cuidado a largo plazo, el Centro de Referencia Estatal Discapacidad y Dependencia (San Andrés del Rabanedo -León-, CRE en adelante). Uno de ellos era usuario de la unidad de estancia diurna del CRE, los demás residían a régimen completo. La tabla 1 describe detalladamente para cada participante los datos demográficos, tipo de discapacidad física, grado de independencia (información del servicio de medicina y fisioterapia del CRE) y nivel de actividad física, el cual se obtuvo preguntando: "en tu día habitual, aparte de la recreación, ¿eres físicamente muy activo, moderadamente activo o sedentario?" (Gretebeck, Ferraro, Black, Holland, y Gretebeck, 2012). La edad media (desviación estándar) de 52.6 (7.7) años superaba el punto de corte de 45 años planteado por Alcedo, Aguado, Arias, González, y Rozada (2008) como indicador de envejecimiento en sujetos con discapacidad. Tres participantes presentaban una discapacidad adquirida (estacionaria en sujetos A y C, progresiva en el sujeto E). Los sujetos B y D tenían una discapacidad congénita. En cuanto a la función física, de acuerdo con el Índice de Barthel (Shah, Vanclay, y Cooper, 1989), el sujeto E respondía a una dependencia severa, los sujetos A y D a una dependencia moderada, el sujeto B a una dependencia escasa y el sujeto C era independiente. Finalmente, sobresalió un perfil sedentario en todos los participantes excepto el sujeto $\mathrm{A}$, quien practicaba ciclismo adaptado con regularidad y había probado el esquí náutico en una ocasión anterior.

Tabla 1. Características de la Muestra de Participantes del Curso de ENI

\begin{tabular}{|c|c|c|c|c|c|}
\hline Sujetos & Sexo & $\begin{array}{l}\text { Edad } \\
\text { (años) }\end{array}$ & Diagnóstico & $\begin{array}{c}\text { Índice de } \\
\text { Barthel }\end{array}$ & $\begin{array}{l}\text { Nivel de actividad } \\
\text { física }\end{array}$ \\
\hline A & $\mathrm{H}$ & 45 & $\begin{array}{l}\text { Lesión cerebelosa focal, trastorno } \\
\text { piramidal y del equilibrio y la } \\
\text { coordinación }\end{array}$ & 80 & Muy activo \\
\hline B & $\mathrm{H}$ & 45 & Parálisis cerebral infantil & 98 & Sedentario \\
\hline $\mathrm{C}$ & M & 54 & TCE, hemiparesia espástica derecha & 100 & Sedentario \\
\hline $\mathrm{D}$ & $\mathrm{H}$ & 56 & Parálisis cerebral infantil & 90 & Sedentario \\
\hline E & $\mathrm{H}$ & 63 & Ataxia de Friedreich & 42 & Sedentario \\
\hline \multicolumn{2}{|c|}{ Media } & 52.6 & & 82.0 & \\
\hline \multicolumn{2}{|c|}{$\mathrm{DE}$} & 7.7 & & 23.7 & \\
\hline
\end{tabular}

Nota. $H=$ hombre; $M=$ mujer; $T C E=$ traumatismo craneoencefálico; $D E=$ desviación estándar 


\section{Instrumentos}

\section{Bienestar subjetivo}

La noción de Bienestar Subjetivo (BS), compuesta por la interrelación de las dimensiones de Satisfacción con la vida, Felicidad y Afectos Positivos y Negativos, es una dimensión importante de la calidad de vida global percibida (Diener y Suh, 1997). Para evaluar el BS en este trabajo, se administró la versión española del Well-being Index (WHO-5) (WHO, 1998), cuestionario muy utilizado internacionalmente y en diversos campos de estudio en relación a poblaciones con discapacidad. Consta de 5 ítems formulados positivamente sobre cómo se ha sentido el sujeto los últimos 14 días en términos de estado de ánimo positivo (buen humor, relajación), vitalidad (sentirse activo y despertarse descansado) e interés general (estar interesado en las cosas). Cada ítem puntúa en una escala Likert desde 0 (nunca) hasta 5 (todo el tiempo). La puntuación final varía entre 0 y 25, pero se recomienda multiplicar esta por 4 para cubrir el espectro del buen bienestar desde 0 (peor bienestar imaginable) hasta 100 (mejor bienestar imaginable) (Topp, Ostergaard, Sondergaard, y Bech, 2015). Este cuestionario ha sido validado mostrando buena consistencia interna (alfa de Cronbach: 0.84) (Bech, Olsen, Kjoller, y Rasmussen, 2003).

\section{Aprendizaje y diversión}

El instrumento del "semáforo" permitió valorar los grados de aprendizaje y diversión de cada sesión. Es válido y apropiado para aplicarse dentro de la dinámica de una clase (Palao y Hernández, 2012), destacando su brevedad y sencillez. Está compuesto por dos preguntas cerradas de triple opción de respuesta (¿Cuánto crees que has aprendido / te has divertido en esta sesión?). El alumno debe elegir un color que responda a su percepción (rojo = poco, amarillo $=$ algo, o verde $=$ mucho) .

\section{Esfuerzo}

Los niveles de percepción subjetiva del esfuerzo (PSE) fueron determinados mediante la escala de Borg (Borg, 1982), cuyo rango oscila entre 6 (no esfuerzo) y 20 (máximo esfuerzo) en una tabla vertical donde los números se acompañan de valoraciones cualitativas sobre el esfuerzo. La escala de PSE de Borg 6-20 es útil para controlar la intensidad del ejercicio en adultos con limitaciones físicas crónicas (Satonaka, Suzuki, y Kawamura, 2012).

\section{Procedimiento}

El curso de ENI fue organizado por el Club Deportivo Esquí Náutico León, entidad con experiencia en la provisión de actividad física adaptada, durante las dos primeras semanas de agosto de 2016. Anteriormente, este club contactó con la dirección del CRE ofreciendo el curso a los usuarios del centro que pudiesen practicar esquí náutico. Además, se informó que se realizaría un estudio de evaluación pre-post como parte del desarrollo del curso y se detallaron sus características. Seis usuarios del CRE expresaron su interés por participar voluntariamente, dando su consentimiento escrito para efectuar cualquier procedimiento, pero solo 5 acudieron al curso por enfermedad sobrevenida de un usuario. El estudio obtuvo la aprobación del Comité (c) Psy, Soc, \& Educ, 2017, Vol. 9(3) 
de Ética de la Universidad de León y se efectuó de acuerdo a la Declaración de Helsinki para investigación biomédica con seres humanos.

El curso de ENI tuvo lugar en el embalse de los Barrios de Luna, entorno de la Reserva de la Bioesfera de Omaña y Luna (León), durante 6 sesiones, una por día. Los participantes se dividieron en dos grupos, de tres sujetos (A, B y E) y dos sujetos ( $\mathrm{C}$ y D). El primer grupo recibió las sesiones $1^{\mathrm{a}}, 3^{\mathrm{a}}$ y $5^{\mathrm{a}}$ (lunes, miércoles y viernes), y el segundo grupo las sesiones $2^{\mathrm{a}}$, $4^{\mathrm{a}}$ y $6^{\mathrm{a}}$ (martes, jueves y lunes). Para que siempre hubiera tres participantes, cada sujeto del primer grupo se acopló al segundo grupo del siguiente modo: sujeto A en $2^{\mathrm{a}}$ sesión, sujeto B en $4^{\mathrm{a}}$ sesión y sujeto $\mathrm{E}$ en $6^{\mathrm{a}}$ sesión. Cada sesión duró cuatro horas aproximadante y tuvo carácter matinal. La enseñanza estuvo dividida en práctica en seco ( 1 hora) y en el agua (2.5-3 horas), a cargo de un piloto y dos instructores de esquí náutico. Tanto en tierra como en el barco, siempre coincidían al menos el piloto, ambos instructores, un participante y un auxiliar del CRE. El curso apostó por un enfoque cooperativo basado en capacidades para optimizar el ambiente de aprendizaje y recurrió a un espectro de inclusión aplicado al esquí náutico, integrado por un conjunto de actividades ajustadas al nivel de cada sujeto para garantizar la igualdad de oportunidades de participación (Suárez-Iglesias y Suárez-García, 2016). Se trataron contenidos teórico-prácticos del esquí náutico para personas con discapacidad como: origen y desarrollo mundial y nacional; qué desafíos experimentan los esquiadores y discusión y evaluación de posibles maneras de afrontarlos; identificación y uso apropiado de equipos deportivos (dos esquís, esquí sentado o Kanski, e hinchable arrastrable) y reconocimiento de otros equipos de asistencia; determinación y análisis de los retos utilizando los equipos deportivos y de asistencia, y descripción y aplicación de formas de cómo superarlos.

El día del inicio del curso (pretest) los participantes completaron en el CRE el cuestionario Well-being Index (WHO-5), así como el día final del curso (postest). El resto de instrumentos de medición del aprendizaje, diversión y esfuerzo fueron administrados en cada sesión, una vez finalizaba y los participantes se encontraban sentados en estado de reposo, habiendo sido familiarizados con su correcto uso durante la enseñanza en seco de las sesiones $1^{\mathrm{a}}$ y $2^{\mathrm{a}}$ del curso.

\section{Análisis de datos}

Las variables son descritas estadísticamente como media aritmética y desviación estándar. La normalidad de las distribuciones fue contrastada con la prueba de Shapiro-Wilk y se aplicó la prueba paramétrica $t$-Student de muestras relacionadas para estudiar el efecto del curso de ENI en el BS, estableciendo el nivel de significación en $\mathrm{p}<0.05$. La eficacia del curso se comprobó estimando el tamaño del efecto mediante la $g$ de Hedges ( $g_{\text {av }}$ para comparaciones intra-sujetos), siguiendo las recomendaciones de Lakens (2013). Se utilizó además el indicador en lenguaje común del tamaño del efecto, que convierte éste en un porcentaje, y expresa en un diseño intra-sujetos la probabilidad de que un sujeto tenga un valor más alto en una medición que en la otra, mejorando así la interpretabilidad de los resultados (Lakens, 2013). Para los distintos análisis se emplearon el paquete estadístico IBM SPSS Statistics 21.0, Microsoft Excel 2013, y la hoja de cálculo de tamaño del efecto de Lakens (2013). 


\section{Resultados}

\section{Bienestar subjetivo}

En relación al BS y atendiendo a la Tabla 2, todos los ítems del cuestionario Well-being Index (WHO-5) presentaron valores altos en el pretest y postest, situándose su media por encima del valor central de la escala de respuesta. Solamente el ítem "4. Me he despertado fresco y descansado" fue inferior al valor 4 en el pretest, siendo el ítem con mayor desviación estándar $(M=3.8 ; D E=1.6)$ y el que obtuvo la puntuación más baja en el postest $(M=4.0 ; D E$ $=1.2$ ). Los ítems que mostraron mayores puntuaciones medias en el postest, así como el mayor porcentaje de cambio positivo pretest-postest fueron "1. Me he sentido alegre y de buen humor" y "5. Mi vida cotidiana ha estado llena de cosas que me interesan", en igual magnitud, seguidos de "2. Me he sentido tranquilo y relajado" (cambios positivos del $14.3 \%$ y $10.0 \%$, respectivamente). Precisamente en los ítems 1 y 5 , los valores de los participantes se comportaron de modo paralelo en el pretest $(M=4.2, D E=0.4)$ y en el postest $(M=4.8, D E=$ $0.4), t_{(4)}=2.45, \mathrm{p}=0.07$, IC $95 \%[-0.08,1.28], g_{\mathrm{av}}$ de Hedges $=1.07$. El lenguaje común del tamaño del efecto indicó que después de controlar las diferencias individuales, la probabilidad de que uno cualquiera de los cinco participantes obtuviese valores más altos para la media del postest que para la media del pretest en los ítems 1 y 5 fue del $86 \%$.

En cuanto al resultado de computar la totalidad de ítems y multiplicar por 4, la puntuación final del cuestionario Well-being Index (WHO-5) fue superior en el postest $(M=89.6, D E=13.1)$ que en el pretest $(M=82.4, D E=8.8), t_{(4)}=2.45, \mathrm{p}=0.07$, IC 95\% $[-0.96,15.36], g_{\text {av }}$ de Hedges $=$ 0.52. El lenguaje común del tamaño del efecto señaló que después de controlar las diferencias individuales, la probabilidad de que uno cualquiera de los cinco participantes obtuviera valores más altos para la media del postest que para la media del pretest fue del $86 \%$.

Tabla 2. Comparaciones Pretest y Postest de Puntuaciones Medias de los Participantes $(n=5)$ según los 5 Ítems del Well-being Index (WHO-5).

\begin{tabular}{|c|c|c|c|c|c|c|}
\hline & $\begin{array}{l}\text { Pretest } \\
\text { M (DE) }\end{array}$ & $\begin{array}{l}\text { Postest } \\
\text { M (DE) }\end{array}$ & $\begin{array}{c}\text { Cambio } \\
(\%)\end{array}$ & $t$ & $\mathrm{p}$ & $\begin{array}{l}g_{\mathrm{av}} \mathrm{de} \\
\text { Hedges }\end{array}$ \\
\hline \multicolumn{7}{|l|}{ "En las últimas dos semanas..." } \\
\hline 1. Me he sentido alegre y de buen humor & $4.2(0.4)$ & $4.8(0.4)$ & 14.3 & 2.45 & 0.07 & 1.07 \\
\hline 2. Me he sentido tranquilo y relajado & $4.0(0.0)$ & $4.4(0.5)$ & 10.0 & 1.63 & 0.18 & 0.83 \\
\hline 3. Me he sentido activo y enérgico & $4.4(0.5)$ & $4.4(0.9)$ & 0.0 & 0.00 & 1.00 & 0.00 \\
\hline 4. Me he despertado fresco y descansado & $3.8(1.6)$ & $4.0(1.2)$ & 5.3 & 1.00 & 0.37 & 0.11 \\
\hline $\begin{array}{l}\text { 5. Mi vida cotidiana ha estado llena de cosas } \\
\text { que me interesan }\end{array}$ & $4.2(0.4)$ & $4.8(0.4)$ & 14.3 & 2.45 & 0.07 & 1.07 \\
\hline Total ítems* & $82.4(8.8)$ & $89.6(13.1)$ & 8.7 & 2.45 & 0.07 & 0.52 \\
\hline
\end{tabular}

Nota. $M=$ media; $D E=$ desviación estándar; $t=$ valor de prueba $t$ para muestras relacionadas; $p=$ prueba de significación, nivel de significación: $\mathrm{p}<0.05 ; g_{\mathrm{av}}$ de Hedges $=$ tamaño del efecto. ${ }^{*}$ Puntuación final multiplicada por 4.

\section{Aprendizaje y diversión}

En la figura 2 se aprecia que los niveles de aprendizaje y diversión percibidos fueron altos. Frente a la diversión percibida, con la mayor media posible en todas las sesiones excepto (c) Psy, Soc, \& Educ, 2017, Vol. 9(3) 
en la $2^{\mathrm{a}}(M=2.7, D E=0.6)$, el aprendizaje percibido presentó valores medios inferiores a 3 en las sesiones $1^{\mathrm{a}}(M=2.3, D E=0.6)$ y $3^{\mathrm{a}}(M=2.7, D E=0.6)$.

\section{Percepción de aprendizaje y diversión}

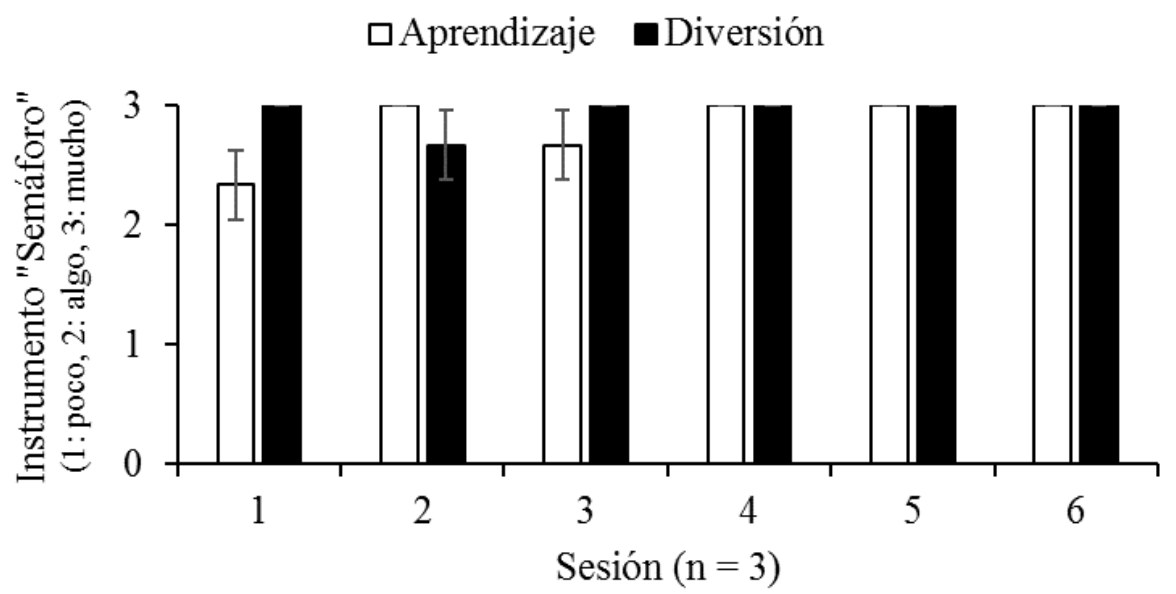

Figura 1. Percepción de aprendizaje y diversión durante las sesiones del curso de ENI.

\section{Esfuerzo}

En la figura 1 se observa que la PSE se encontraba en la mayoría de sesiones en niveles entre "bastante ligero" y "algo duro", alcanzando un nivel cercano a "duro" solo en la $5^{\text {a }}$ sesión $(M=14.7, D E=3.1)$, mientras que descendió al nivel "muy ligero" en la $6^{a}$ sesión $(M=9.0$, $D E=3.5)$. La PSE del total de sesiones arrojó de media una intensidad que puede considerarse moderada $(M=12.2, D E=3.1)$.

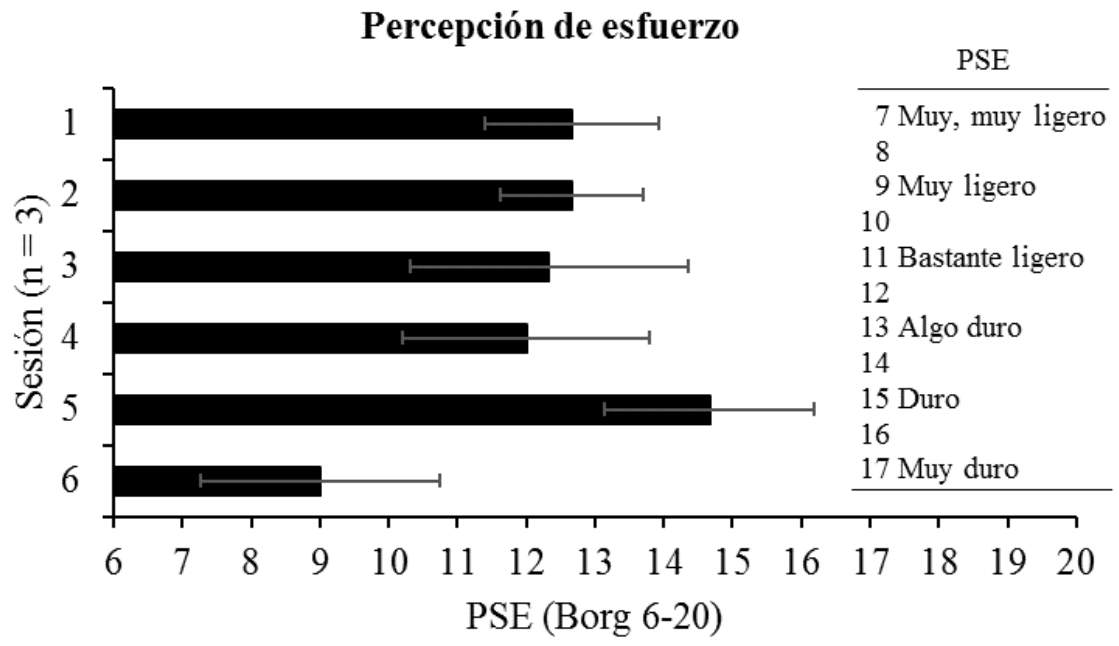

Figura 2. Percepción subjetiva del esfuerzo (PSE) durante las sesiones del curso de ENI.

\section{Discusión}

El objetivo principal de este trabajo ha sido analizar el impacto sobre el BS percibido de cinco adultos que envejecen con discapacidades físicas participantes en un curso de ENI, así 
como identificar su percepción sobre el aprendizaje, diversión y esfuerzo de las seis sesiones del curso.

Al comprobar los cambios en el BS de los participantes terminado el curso, el tamaño del efecto ( $g$ de Hedges de 0.52) está en línea con los cambios beneficiosos observados por Bowler et al. (2010) para medidas de bienestar psicológico, en una revisión sistemática de estudios que comparan el antes y el después de llevar a cabo una actividad en un ambiente natural. No obstante, los cambios positivos reportados para sensaciones de energía, tranquilidad y fatiga ( $g$ de Hedges de $0.76,0.07$ y 0.76 , respectivamente), parecen contrastar con nuestros tamaños del efecto para las mismas sensaciones del cuestionario Well-being Index (WHO-5), en concreto, el ítem " 3 . Me he sentido activo y enérgico" $\left(g_{\mathrm{av}}\right.$ de Hedges $\left.=0.00\right)$, el ítem " 2 . Me he sentido tranquilo y relajado" $\left(g_{\text {av }}\right.$ de Hedges $\left.=0.83\right)$ y el ítem " 4 . Me he despertado fresco y descansado" ( $g_{\mathrm{av}}$ de Hedges $\left.=0.11\right)$; si bien los participantes más comunes de los estudios manejados por Bowler et al. (2010) eran estudiantes universitarios y personas físicamente activas, en contraposición a los adultos sedentarios que envejecen con limitaciones físicas de nuestro estudio. Además, Bowler et al. (2010) advertían que tratándose de condiciones de salud específicas, los tamaños del efecto merecían una investigación más profunda. En este sentido, Pasanen et al. (2014) indagaron sobre la relación entre practicar actividades recreativas en la naturaleza y la salud general percibida y el bienestar emocional, en base a una encuesta nacional a población finlandesa, informando que aquellos con discapacidades crónicas u otros obstáculos para ejercitarse al aire libre veían reducido el efecto positivo de dicha relación, tal y como ocurre para los ítems 3 y 4.

También referente al BS y en nuestros participantes, se observan tamaños del efecto de gran magnitud ( $g_{\mathrm{av}}$ de Hedges $=1.07$ ) en dos ítems, el "1. Me he sentido alegre y de buen humor" y el "5. Mi vida cotidiana ha estado llena de cosas que me interesan", en consonancia con las emociones de placer y felicidad experimentadas después de la práctica de surf adaptado (Lopes, 2015). Conforme al modelo de Newman, Tay, y Diener (2014) sobre los mecanismos psicológicos que actúan como mediadores del BS en el ocio, los potenciales factores que explican el cambio positivo en los ítems mencionados serían: a) la "afiliación", dado el carácter social del curso; b) el "desprendimiento-recuperación", al romper la rutina diaria del centro de cuidado a largo plazo mediante la práctica novedosa y excitante de esquí náutico; y c) la "maestría", porque la metodología y contenidos del curso permitieron a los participantes superar desafíos y mejorar gradualmente sus habilidades.

Por otro lado, los beneficios para el BS de los participantes en actividades al aire libre dependen de cuánto se aprende y los vínculos establecidos entre lo aprendido y otras áreas de la vida (O’Brien et al., 2011). Atendiendo a nuestros resultados, la percepción de haber aprendido mucho en más de la mitad de las sesiones puede estar causada por el enfoque basado en capacidades del curso (Suárez-Iglesias y Suárez-García, 2016), que compatibilizó las demandas de aprendizaje y las capacidades funcionales del participante, el cual adoptó junto al instructor un rol de co-aprendizaje a la hora de decidir la tipología de las actividades y los objetivos de la sesión. Otra razón para los destacados niveles de aprendizaje podría ser que el curso es una actividad náutica $\mathrm{y}$, como tal, es rica en contenidos que promueven la educación integral: aspectos afectivos, cognitivos, motores, sociales (Peñarrubia, Guillén, y Lapetra, 2016). El curso de ENI incluía varios de estos aspectos, concretados en contenidos téorico-prácticos de elección, evaluación y retroinformación, habilidades de equilibrio dinámico o procesos de 
cooperación (Suárez-Iglesias y Suárez-García, 2016). Su desarrollo en el medio natural también pudo incrementar el aspecto educativo al favorecer el aprendizaje de nuevas habilidades en paralelo al conocimiento del entorno (Palmi y Martín, 1997).

Cabe destacar que los participantes aseguraron haberse divertido mucho en cinco de las seis sesiones del curso. Éste siguió un enfoque basado en capacidades, que equilibra las demandas de la actividad y las destrezas del practicante y permite el éxito en la práctica, lo que puede explicar el elevado disfrute (Palmi y Martín, 1997). Adicionalmente, la exposición al entorno agradable y natural del embalse pudo convertir la actividad en potencialmente más divertida, al reducir la conciencia sobre las sensaciones fisiológicas y emociones negativas y disminuir en consecuencia los niveles de percepción de esfuerzo (Gladwell et al., 2013).

La PSE de más del 50\% de sesiones, oscilando en niveles de "bastante ligero" a "algo duro", es coherente con este planteamiento. Esta intensidad moderada promedio, ideal para la eficacia de los programas de actividad física entre los que envejecen con limitaciones de movimiento (Rosenberg et al., 2011), puede incluso estar motivada por la intermitencia de la práctica de esquí náutico, y es que los periodos de descanso y actividad alternativos son útiles para minimizar las sensaciones de fatiga en personas con discapacidad (Cooper et al., 1999).

En este trabajo, el pequeño tamaño muestral dificulta la generalización de los resultados, pero la muestra es representativa del único curso intensivo de esquí náutico para personas con discapacidad celebrado en España en 2016. Otra limitación es que la intensidad del ejercicio fue valorada únicamente con la PSE. Sin embargo, Satonaka et al. (2012) recomiendan el uso conjunto de variables fisiológicas, como frecuencia cardiaca, en población inactiva con discapacidades físicas crónicas.

En síntesis, nuestro hallazgo fundamental es que cursos intensivos de ENI pueden ser pertinentes como AFTL en la promoción general del BS de las personas que envecejen con limitaciones de movimiento. Aunque resultarían necesarios otros estudios que precisen los mecanismos que promueven el BS en esta clase de intervenciones, los cambios positivos en nuestro trabajo vinieron acompañados de sesiones de ENI con percepciones de alto aprendizaje y diversión y un esfuerzo moderado. Teniendo en cuenta que la salud y la diversión son facilitadores personales en la participación deportiva para personas con discapacidades físicas (Jaarsma, Dijkstra, Geertzen, y Dekker, 2014), el esquí náutico podría emplearse como estrategia viable para la participación en AFTL de adultos con discapacidades, contribuyendo al concepto de envejecimiento activo y a su inclusión social (Pagán-Rodríguez, 2014), en pos de una mejor calidad de vida.

\section{Referencias}

Alcedo, M. A., Aguado, A., Arias, B., González, M., y Rozada, C. (2008). Escala de Calidad de Vida (ECV) para personas con discapacidad que envejecen: estudio preliminar. Psychosocial Intervention, 17(2), 153-167.

Barton, J., y Pretty, J. (2010). What is the best dose of nature and green exercise for improving mental health? A multi-study analysis. Environmental Science \& Technology, 44(10), 3947-3955. 
Bech, P., Olsen, L. R., Kjoller, M., y Rasmussen, N. K. (2003). Measuring well-being rather than the absence of distress symptoms: a comparison of the SF-36 Mental Health subscale and the WHO-Five Well-Being Scale. International Journal of Methods in Psychiatric Research, 12(2), 85-91.

Borg, G. (1982). Psychophysical bases of perceived exertion. Medicine and Science in Sports and Exercise, 14(5), 377-381.

Bowler, D. E., Buyung-Ali, L. M., Knight, T. M., y Pullin, A. S. (2010). A systematic review of evidence for the added benefits to health of exposure to natural environments. BMC Public Health, 10, 456. http://doi.org/10.1186/1471-2458-10-456

Cooper, R. A., Quatrano, L. A., Axelson, P. W., Harlan, W., Stineman, M., Franklin, B., ... Painter, P. (1999). Research on physical activity and health among people with disabilities: a consensus statement. Journal of Rehabilitation Research and Development, 36(2), 142-154.

Diener, E. D., y Suh, E. (1997). Measuring quality of life: economic, social, and subjective indicators. Social Indicators Research, 40(1/2), 189-216.

Drakou, A., De Vreese, R., Lofthus, T., y Muscat, J. (2011). Motivating people to be physically active in green spaces. In K. Nilsson, M. Sangster, C. Gallis, T. Hartig, S. de Vries, K. Seeland, \& J. Schipperijn (Eds.), Forests, Trees and Human Health (pp. 283-306). Dordrecht: Springer Netherlands. http://doi.org/10.1007/978-90-481-9806-1_10

Gladwell, V. F., Brown, D. K., Wood, C., Sandercock, G. R., y Barton, J. L. (2013). The great outdoors: how a green exercise environment can benefit all. Extreme Physiology \& Medicine, 2(1), 3. http://doi.org/10.1186/2046-7648-2-3

Gretebeck, R. J., Ferraro, K. F., Black, D. R., Holland, K., y Gretebeck, K. A. (2012). Longitudinal change in physical activity and disability in adults. American Journal of Health Behavior, 36(3), 385-394. http://doi.org/10.5993/AJHB.36.3.9

Hutzler, Y. (2003). Attitudes toward the participation of individuals with disabilities in physical activity: a review. Quest, 55(4), 347-373.

Hutzler, Y., y Sherrill, C. (2007). Defining adapted physical activity: international perspectives. Adapted Physical Activity Quarterly, 24(1), 1-20.

Jaarsma, E. A., Dijkstra, P. U., Geertzen, J. H. B., y Dekker, R. (2014). Barriers to and facilitators of sports participation for people with physical disabilities: a systematic review. Scandinavian Journal of Medicine \& Science in Sports, 24(6), 871-881.

Lakens, D. (2013). Calculating and reporting effect sizes to facilitate cumulative science: a practical primer for t-tests and ANOVAs. Frontiers in Psychology, 4(Nov), 1-12.

Lopes, J. T. (2015). Adapted surfing as a tool to promote inclusion and rising disability awareness in Portugal. Journal of Sport for Development, 3(5), 4-10.

Lundberg, N., Bennett, J., y Smith, S. (2011). Outcomes of adaptive sports and recreation participation among veterans returning from combat with acquired disability. Therapeutic Recreation Journal, 45(2), 105-120.

Malone, L. A., Barfield, J. P., y Brasher, J. D. (2012). Perceived benefits and barriers to exercise among persons with physical disabilities or chronic health conditions within action or maintenance stages of exercise. Disability and Health Journal, 5(4), 254-260.

Martin Ginis, K. A., Ma, J. K., Latimer-Cheung, A. E., y Rimmer, J. H. (2016). A systematic review of review articles addressing factors related to physical activity participation among children and adults with physical disabilities. Health Psychology Review, 10(4), 478-494. 
Newman, D. B., Tay, L., y Diener, E. (2014). Leisure and subjective well-being: a model of psychological mechanisms as mediating factors. Journal of Happiness Studies, 15(3), 555578.

O’Brien, L., Burls, A., Bentsen, P., Hilmo, I., Holter, K., Haberling, D., ... McLoughlin, J. (2011). Outdoor education, life long learning and skills development in woodlands and green spaces: the potential links to health and well-being. In J. S. Kjell Nilsson, Marcus Sangster, Christos Gallis, Terry Hartig, Sjerp de Vries, Klaus Seeland (Ed.), Forests, Trees and Human Health (pp. 343-372). Dordrecht: Springer Netherlands. http://doi.org/10.1007/97890-481-9806-1 12

Pagán-Rodríguez, R. (2014). How do disabled individuals spend their leisure time? Disability and Health Journal, 7(2), 196-205.

Palao, J. M., y Hernández, E. (2012). Validación de un instrumento para valorar la percepción del aprendizaje y el nivel de diversión del alumno en educación física. El semáforo. Cuadernos de Psicología Del Deporte, 12(1), 25-32.

Palmi, J., y Martín, A. (1997). Las actividades físico-deportivas en el medio natural y sus efectos sobre la salud y la calidad de vida: factores psicológicos asociados. Revista de Psicología Del Deporte, 6(2), 147-160.

Pasanen, T. P., Tyrväinen, L., y Korpela, K. M. (2014). The relationship between perceived health and physical activity indoors, outdoors in built environments, and outdoors in nature. Applied Psychology: Health and Well-Being, 6(3), 324-346.

Peñarrubia, C., Guillén, R., y Lapetra, S. (2016). Las actividades en el medio natural en Educación Física, ¿teoría o práctica? Cultura, Ciencia Y Deporte, 11(31), 27-36.

Rosenberg, D. E., Bombardier, C. H., Hoffman, J. M., y Belza, B. (2011). Physical activity among persons aging with mobility disabilities: shaping a research agenda. Journal of Aging Research, 2011, 708510. http://doi.org/10.4061/2011/708510

Satonaka, A., Suzuki, N., y Kawamura, M. (2012). Ratings of perceived exertion in adults with chronically physical challenges. The Journal of Sports Medicine and Physical Fitness, 52(5), 474-482.

Shah, S., Vanclay, F., y Cooper, B. (1989). Improving the sensitivity of the Barthel Index for stroke rehabilitation. Journal of Clinical Epidemiology, 42(8), 703-709.

Suárez-Iglesias, D., y Suárez-García, M. (2016). Esquí náutico como medio de inclusión social de personas con discapacidad: un enfoque basado en capacidades en torno a un espectro de inclusión. In J. Gallego, M. Alcaraz-Ibáñez, J. M. Aguilar-Parra, A. J. Cangas, \& D. Martínez Luque (Eds.), Avances en la actividad física y deportiva inclusiva (pp. 102-121). Almería: Editorial Universidad de Almería.

Topp, C. W., Ostergaard, S. D., Sondergaard, S., y Bech, P. (2015). The WHO-5 Well-Being Index: a systematic review of the literature. Psychotherapy and Psychosomatics, 84(3), $167-176$.

WHO. (1998). Wellbeing measures in primary health care/The DepCare project: report on a WHO meeting, Stockholm, Sweden 12-13 February, 1998. Copenhagen. Retrieved from http://www.euro.who.int/_data/assets/pdf_file/0016/130750/E60246.pdf 